\title{
Theoretical Investigation of Controlled Generation of a Dense Attosecond Relativistic Electron Bunch from the Interaction of an Ultrashort Laser Pulse with a Nanofilm
}

\author{
Victor V. Kulagin, ${ }^{1, *}$ Vladimir A. Cherepenin, ${ }^{2}$ Min Sup Hur, ${ }^{1}$ and Hyyong Suk ${ }^{1, \dagger}$ \\ ${ }^{1}$ Center for Advanced Accelerators, KERI, Changwon, 641-120, Republic of Korea \\ ${ }^{2}$ Institute of Radioengineering and Electronics RAS, Mohovaya 11, Moscow, 125009, Russia
}

(Received 18 September 2006; published 21 September 2007)

\begin{abstract}
For controllable generation of an isolated attosecond relativistic electron bunch [relativistic electron mirror (REM)] with nearly solid-state density, we propose using a solid nanofilm illuminated normally by an ultraintense femtosecond laser pulse having a sharp rising edge. With two-dimensional (2D) particlein-cell (PIC) simulations, we show that, in spite of Coulomb forces, all of the electrons in the laser spot can be accelerated synchronously, and the REM keeps its surface charge density during evolution. We also developed a self-consistent 1D theory, which takes into account Coulomb forces, radiation of the electrons, and laser amplitude depletion. This theory allows us to predict the REM parameters and shows a good agreement with the 2D PIC simulations.
\end{abstract}

DOI: 10.1103/PhysRevLett.99.124801

PACS numbers: 41.75.Jv, 41.75.Ht, 52.38.Kd

Generation of attosecond relativistic electron beams is of great importance for modern physics. These beams can be used in modern laser-wakefield accelerators and freeelectron lasers for injection, in attosecond electron diffraction and microscopy, in generation of ultrashort coherent $\mathrm{x}$ ray radiation via Thomson scattering, and in many other applications, providing time-resolved studies in physics, biology, chemistry, etc., with the attosecond time-scale resolution. The main requirement for attosecond electron bunches is the controllability of their parameters, including length, charge, and energy.

In high-density (overcritical) plasmas, two mechanisms for generation of ultrashort electron beams - the $\mathbf{v} \times \mathbf{B}$ heating and the vacuum heating - were investigated recently [1-4]. The length of the electron beam is about the laser pulse length here with a wide energy spread for electrons; in addition, the beam parameters are difficult to control. In low-density (underdense) plasmas, a single electron bunch can be generated by laser-wakefield acceleration mechanism [5-7] but the length of the bunch is not shorter than 1-5 $\mu \mathrm{m}$ (several femtoseconds). In a vacuum, a single ultrashort electron beam can be generated through laser compression of a longer electron beam [8,9]; however, the charge of the bunch here is considerably smaller than $1 \mathrm{pC}$. The same compression can be applied for thin (1 $\mu \mathrm{m}$ and less) plasma layers of low (gas) density [10$12]$, but the practical realization of such layers is under question now.

In this Letter, we propose using a nanofilm (film with a thickness of $10 \mathrm{~nm}$ or less) as a solid-state density target for generation of an attosecond relativistic electron bunch. It is shown that, when this target is irradiated normally by a superhigh intensity laser pulse with a sharp rising edge (nonadiabatic laser pulse), all electrons of the plasma layer can achieve relativistic longitudinal velocities synchronously when the dimensionless field amplitude becomes large enough, $a_{0} \gg \alpha\left[a_{0}=|e| E_{0} /(m c \omega), \alpha=\right.$ $\pi\left(\omega_{p}^{2} / \omega^{2}\right)(l / \lambda)$, where $e$ and $m$ are the charge and the mass of an electron, $c$ is the speed of light, $E_{0}, \omega$, and $\lambda$ are the amplitude, the frequency, and the wavelength of the laser field in a vacuum, $\omega_{p}=\sqrt{4 \pi n_{0} e^{2} / m}$ is the characteristic plasma frequency, $n_{0}$ and $l$ are the density and the thickness of the nanofilm]. Our scheme has the following features: (i) only a single electron bunch of attosecond length is generated, (ii) it can generate a very large charge $(>10 \mathrm{nC})$, and (iii) its parameters can be controlled easily. We call such a bunch a relativistic electron mirror (REM). In this Letter, we demonstrate for the first time the feasibility of generation of such an attosecond electron bunch by using virtual experiments, i.e., 2D particle-in-cell (PIC) simulations. We revealed the new effect of "charge freezing," consisting in a slow (in a time-scale of longitudinal acceleration) removal of the electrons from the central part of the REM, even when the laser beam diameter is not extremely large, that allows for the REM to keep initial surface charge density during its entire lifetime. Furthermore, we developed a new self-consistent 1D theory to explain the acceleration process. We found that laser field depletion, strong radiation of the accelerated electrons, and high radiation friction play very crucial roles in the acceleration process in the solidlike high-density plasma so our theory includes all of these effects. This theory allows us to calculate all parameters of the REM electrons, i.e., trajectories, momenta, energies, etc., and turns out to be in good agreement with the 2D PIC simulations.

The main condition for realization of the proposed generation scheme is the availability of nonadiabatic laser pulses. Recently, few-cycle laser pulses with very sharp fronts have been generated experimentally $[13,14]$ and, in the near future, one can expect the generation of few-cycle laser pulses of petawatt and even exawatt $\left(10^{18} \mathrm{~W}\right)$ levels [15]. 
To demonstrate the generation of the REM, we performed 2D PIC simulations with the OSIRIS code [16] for linearly $y$-polarized laser pulses $(\lambda=1 \mu \mathrm{m})$, running along the $z$ axis in the positive direction and having two different beam waists $w_{0}$ at focus: $k w_{0}=50$ (laser spot size is $2 w_{0} \approx 16 \lambda$ ) and $k w_{0}=12.5$ (laser spot size is about $4 \lambda$ ), where $k=2 \pi / \lambda$. To reduce the Coulomb expansion of the REM, one needs to decrease $\alpha$, which is proportional to the surface density of electrons $n_{0} l[10-$ 12,17]. So we used a nanofilm (positioned at the beam focus) with a thickness of $10 \mathrm{~nm}$ (routinely available in experiment [18]) and with a reasonable solid-state density $n_{0}=318.3 n_{\text {cr }}$, where $n_{\text {cr }}$ is the critical density ( $\alpha$ is $\left.\sim 10\right)$. To ensure the validity of inequality $a_{0} \gg \alpha$, a laser pulse with a Gaussian transverse profile and a steplike longitudinal envelope with the amplitude $a_{0}=60$ was used. In the simulation, we used 630 grid points per $\lambda$ along the longitudinal direction and $16\left(k w_{0}=50\right)$ or $63\left(k w_{0}=12.5\right)$ grid points per $\lambda$ along the transverse direction, and 256 particles per cell were employed. The plasma was assumed to be preionized (for $a_{0}=60$, a delay in ionization is small enough to cause no effect if the target material has not very high nucleus charge $Z<10$ ) and collisionless with the ions mass $m_{i}=3671 m$.

Phase spaces $k z-k y$ and $p_{z}-k y$ (the momentum $p_{z}$ is normalized by $m c$ ) are presented for two different times $\omega t=13$ and $\omega t=20$ in Fig. $1\left(k w_{0}=50\right)$. The electrons near the laser beam axis $(k y=100)$ form the REM. Because of a snowplow action of the laser pulse [1012,19], all electrons are removed from the central part of the nanofilm and are accelerated longitudinally to relativistic velocities. Because of large Coulomb forces, the electrons in the left side of the REM (called left electrons below) turn back at some time while other electrons continue to move to the right. Thus, left electrons on the laser beam axis turn back at about $\omega t \simeq 24$ (cf. Fig. 2); this time can be defined as a lifetime $\tau_{l}$ of the REM (for the laser waist $k w_{0}=12.5$, the lifetime is about $\tau_{l} \simeq 23$ ). The position of the first turning point of the left electrons depends primarily on the parameters $a_{0}$ and $\alpha$. Therefore, because of the Gaussian transverse profile of the laser pulse, the diameter of the REM gradually decreases with time (for $\omega t \simeq \tau_{l}$, the diameter of the REM is about zero). However, even after the turn of all left electrons, the major part of the electrons continues to move, forming partial REM with the full thickness of about $\lambda$. On the edges of the REM, the laser pulse produces usual heating of the plasma (e.g., near $k y=50$ and $k y=150$ in Fig. 1). During acceleration, the REM "eats away" the first half-cycle of the laser pulse (amplitude transmission is less than 10\%) so by the end of the REM lifetime, the first half-cycle of the laser pulse almost totally disappears. The phase spaces for $k w_{0}=$ 12.5 (not shown here) are very similar to those presented in Fig. 1, with the only important difference being the fourfold decrease of the REM dimension along the $y$ axis. This means that the considered process is effectively quasi-one-dimensional and regular.


FIG. 1 (color online). Phase spaces $k z-k y(\mathrm{a}),(\mathrm{c})$ and $p_{z}-$ $k y$ (b), (d) for the electrons accelerated by the laser pulse having the waist $k w_{0}=50$ for $\omega t=13$ (upper panels) and $\omega t=20$ (lower panels).

The coordinates of the leftmost and the rightmost electrons of the REM as functions of time are shown in Fig. 2. The full thickness of the REM in the central part is considerably smaller than $\lambda$ (see also Fig. 3) right up to the first turning point of the left electrons, where it is about $\lambda / 2$ $(k l \simeq \pi)$. The full thickness of the REM near $\omega t \simeq 13$ (which is the best time for use of the REM since the momenta $p_{z}$ of the electrons reach maxima) is only about $\lambda / 10$ (cf. insets in Fig. 2), i.e., about 300 attoseconds (the full length at half maximum here is only about 90 attoseconds). Also, the REM remains strongly overcritical throughout the entire lifetime.

The full thickness $k l$ and the surface charge density $\alpha_{c}$ of the REM as functions of the transverse coordinate $y$ are presented in Fig. $3\left(k w_{0}=50\right)$. It is remarkable that, during the evolution, the surface charge density $\alpha_{c}$ of the REM remains close to the initial value $\alpha=10$ for the nanofilm, and there is almost no charge removal from the central part of the REM to the peripheral parts even for a moderate diameter of the laser beam. We call this feature a charge freezing effect of the superintense nonadiabatic laser pulse, as opposed to the well-known drilling effect of an adiabatic laser pulse. Also, inside the REM, $k l$ and $\alpha_{c}$ change smoothly indicating the coherence of the electron movements contrary to the outer regions, where the electrons move chaotically and $\alpha_{c}$ changes very rapidly. Again, 


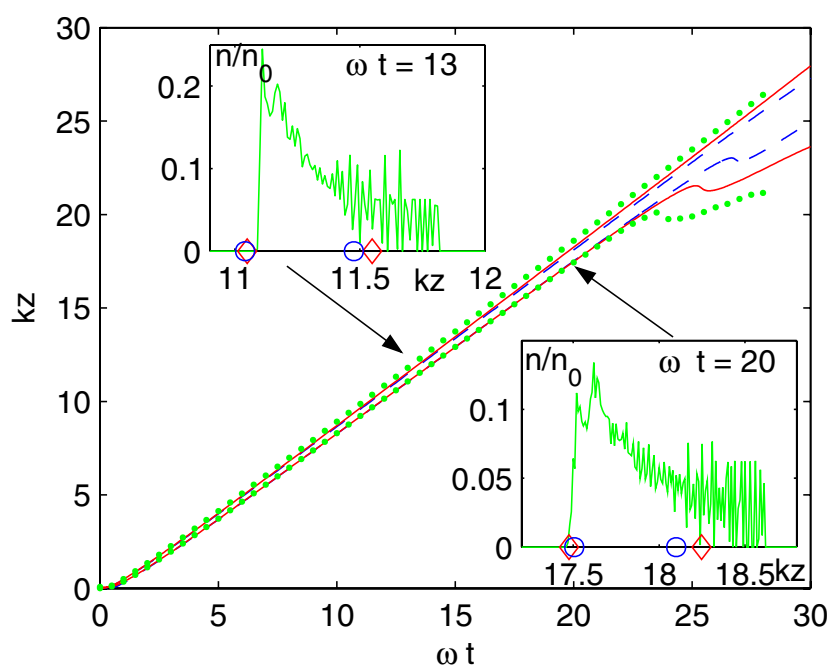

FIG. 2 (color online). Coordinates of the leftmost and the rightmost electrons of the REM as functions of time: dots (green online) are the results for the 2D PIC simulations with $k w_{0}=50$ taken on the laser beam axis $(k y=100)$, solid lines and diamonds (both red online) are the numerical solutions of the system (3), and dashed lines and circles (both blue online) are the approximate analytical solutions according to Eqs. (4). In the insets, the electron densities (normalized by the initial density $n_{0}$ ) on the laser beam axis for $\omega t=13$ and $\omega t=20$ are presented (green solid lines online).

the same features are characteristic for the smaller beam size $k w_{0}=12.5$ (not shown here), and the graphics are very similar with rescaling of the ky axis by 4 times.

So the REM is a transient coherent structure, in which all electrons move synchronously. It exists only for a limited period of time. In the above simulations, the lifetime of the REM is about 4 laser periods (13 femtoseconds). Larger values for $a_{0}$ or smaller values for $\alpha$ can increase the lifetime of the REM up to hundreds of femtoseconds [12].

Let us now elaborate the 1D theory for the generation of the REM. The nanofilm will be modeled with a set of electron sheets (ESs), each numbered by its initial coordinate $z_{0} \in[0, l]$. The laser field will be modified by virtue of an interference with radiation emitted by these ESs, and the motion of the ESs will be defined by the combined electromagnetic field (the self-consistent model is necessary here since $\alpha>1[10,12])$. The fields of one ES are determined by 1D analogs of the classical Lienard-
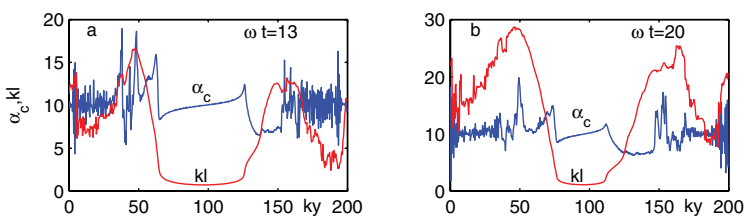

FIG. 3 (color online). Thickness $k l$ (light gray; red online) and surface charge density $\alpha_{c}$ (dark gray; blue online) for the REM formed by the laser pulse with the waist $k w_{0}=50$ [(a) $\omega t=13$ and (b) $\omega t=20]$.
Wiechert solutions $[10,12]$. Inserting the sum of the radiation fields of ESs into equations of motion for the ES with the coordinate $z_{0}$, we will obtain the nonlinear integrodifferential equation with delay [12], which contains the ES variables only. In general cases, the solution should be computed numerically for a large number of ESs simultaneously to evaluate the integrals.

To reduce the system, we will use in this Letter the following simplifying assumptions. In the initial coherent stage of the nanofilm's evolution, the thickness of the nanofilm is small, $l \ll \lambda$, and there is neither heating of the nanofilm nor turbulent motion of the ESs. Then, we assume that the radiation field of each ES immediately acts on the other ESs and there are no intersections of trajectories of the ESs. Besides, we assume that the ESs are immediately removed from the ion layer after beginning of the interaction since $a_{0} \gg \alpha$ and $l \ll \lambda$. Then, we obtain for the ES with the initial coordinate $z_{0}$

$$
\begin{aligned}
d p_{y} / d(\omega t)= & -a_{0} e_{y} \kappa / \gamma \\
& -\alpha\left[\kappa I_{1} / \gamma+\left(1+p_{y}^{2}\right) I_{2} /(\kappa \gamma)\right], \\
d \kappa / d(\omega t)= & 2 \alpha\left[\kappa\left(1-z_{0} / l\right)-p_{y} I_{2}\right] / \gamma,
\end{aligned}
$$

where $e_{y}=\sin (\omega t-k z), \boldsymbol{p}=\gamma \mathbf{v} / c$ is the normalized momentum of the electrons with velocity $\mathbf{v}, \quad \gamma=$ $\left(\sqrt{1-v^{2} / c^{2}}\right)^{-1}$,

$$
\begin{aligned}
& I_{1}\left(z_{0}, t\right)=l^{-1} \int_{0}^{z_{0}} \frac{p_{y}\left(z_{0}^{\prime}, t\right) d z_{0}^{\prime}}{\kappa\left(z_{0}^{\prime}, t\right)}, \\
& I_{2}\left(z_{0}, t\right)=l^{-1} \int_{z_{0}}^{l} \frac{p_{y}\left(z_{0}^{\prime}, t\right) \kappa\left(z_{0}^{\prime}, t\right) d z_{0}^{\prime}}{1+p_{y}^{2}\left(z_{0}^{\prime}, t\right)},
\end{aligned}
$$

and we introduce the parameter $\kappa=\gamma-p_{z}$ [as usual, $\gamma=$ $\left.\left(1+p_{y}^{2}+\kappa^{2}\right) /(2 \kappa), \quad p_{z}=\left(1+p_{y}^{2}-\kappa^{2}\right) /(2 \kappa)\right]$. Physically, the integral $I_{1}$ describes the action of the radiation field of all left ESs on the ES with coordinate $z_{0}$, and $I_{2}$ describes the action of the radiation field of right ESs.

To evaluate the integrals $I_{1}$ and $I_{2}$, we suppose that the integrands in Eqs. (2) can be represented in any internal point $z_{0} \in[0, l]$ as a linear interpolation of their values at points $z_{0}=0$ and $z_{0}=l$. Then, $\kappa_{l}=1$ and

$$
\begin{aligned}
\frac{d p_{y 0}}{d(\omega t)} & =-a_{0} e_{y} \frac{\kappa_{0}}{\gamma_{0}}-\frac{\alpha}{2 \gamma_{0}}\left(p_{y 0}+\frac{p_{y l}}{\kappa_{0}} \frac{1+p_{y 0}^{2}}{1+p_{y l}^{2}}\right), \\
\frac{d \kappa_{0}}{d(\omega t)} & =\frac{\alpha \kappa_{0}}{\gamma_{0}}\left[\frac{1}{1+p_{y 0}^{2}}+1-\frac{p_{y 0} p_{y l}}{\kappa_{0}\left(1+p_{y l}^{2}\right)}\right], \\
\frac{d p_{y l}}{d(\omega t)} & =-\frac{a_{0} e_{y}}{\gamma_{l}}-\frac{\alpha}{2 \gamma_{l}}\left(p_{y l}+\frac{p_{y 0}}{\kappa_{0}}\right),
\end{aligned}
$$

where indexes 0 and $l$ stand for the left $\left(z_{0}=0\right)$ and the right $\left(z_{0}=l\right)$ ESs. So equations of motion of all ESs are reduced now to the coupled nonlinear system of equations for the two outer ESs, which can be easily solved numerically. 
To uncouple Eqs. (3) and to get the approximate analytical solutions, we suppose that $p_{y 0} / \kappa_{0}=p_{y l} / \kappa_{l}=p_{y l}$ in the large parentheses on the right-hand sides of Eqs. (3) (i.e., in terms originating from the self-action of the own radiation of the nanofilm). After decoupling, we transfer to new independent variables $\theta_{0}=\omega t-k Z_{0}$ and $\theta_{l}=\omega t-$ $k Z_{l}$, where $Z_{0}$ and $Z_{l}$ are the $z$ coordinates of the right and the left ESs. The parameter $\kappa_{0}$ is a slow function so the equation for $p_{y 0}$ can be approximately solved supposing $\kappa_{0}=$ const $\ll\left|p_{y 0}\right|$. Substituting approximate solution $p_{y 0}=a_{0} \kappa_{0} \alpha^{-1} \sin \theta_{0}$ into the equation for $\kappa_{0}$ and integrating this equation by $\theta_{0}$ over the period of the laser field to remove the $\theta_{0}$ dependence, we eventually obtain the following approximate parametric solutions for Eqs. (3) with parameters $\theta_{0}$ and $\theta_{l}$

$$
\begin{gathered}
\kappa_{0}=1+\alpha^{2} \theta_{0} / a_{0}+\ln \left(1+\alpha^{2} \theta_{0} / a_{0}\right), \\
p_{y 0}=-a_{0} \kappa_{0}\left(\kappa_{0}^{2}+\alpha^{2}\right)^{-1}\left\{\alpha \sin \theta_{0}-\kappa_{0}\left[\cos \theta_{0}\right.\right. \\
\left.\left.-\exp \left(-\alpha \theta_{0} / \kappa_{0}\right)\right]\right\}, \\
k Z_{0}=\int_{0}^{\theta_{0}}\left(1+p_{y 0}^{2}\right) d \theta_{0}^{\prime} /\left(2 \kappa_{0}^{2}\right)-\theta_{0} / 2, \\
p_{y l}=-a_{0}\left[\alpha \sin \theta_{l}-\cos \theta_{l}+\exp \left(-\alpha \theta_{l}\right)\right] /\left(1+\alpha^{2}\right), \\
k Z_{l}=\int_{0}^{\theta_{l}} p_{y l}^{2} d \theta_{l}^{\prime} / 2, \quad \omega t=\theta_{0}+k Z_{0}=\theta_{l}+k Z_{l},
\end{gathered}
$$

The solutions for Eqs. (3) and (4) coincide well with the PIC results almost until the first turning point of the left ES at $\omega t \simeq 25$ (cf. Fig. 2). From the insets of Fig. 2, one can conclude that the analytical approach describes well the main peak of the electron density discarding only the lowdensity part of the REM. So the elaborated analytical approach [the reduced system (3) and the approximate solutions of this system (4)] can be used for quick calculation of the dynamical parameters of the REM.

Now, let us take examples. The laser power for $k w_{0}=$ 50 and $a_{0}=60$ amounts to $5 \mathrm{PW}$, and the charge of the REM (estimated inside $2 w_{0}$ spot) is $160 \mathrm{nC}\left(10^{12}\right.$ electrons). For $k w_{0}=12.5$ the values are more modest: $300 \mathrm{TW}$ and $10 \mathrm{nC}\left(6.25 \times 10^{10}\right.$ electrons $)$, and this power can already be realized nowadays. For the proof-ofprinciple experiments, using, e.g., graphene sheets [20] as a target $(l=2 \mathrm{~nm}, \alpha \simeq 2)$ and with a laser spot size of about $w_{0} \simeq \lambda$, the laser power of $2 \mathrm{TW}$ is enough. If the laser pulse has a smooth front, then the electrons of the REM remain motionless in the longitudinal direction until the laser amplitude reaches the value $a_{0} \sim \alpha[12,17]$. After that, the REM will be formed by the first half-cycle, which has the amplitude $a_{0 f}$ larger than $\alpha$. So for the generation of a good REM by a smooth laser pulse, the high peak-topeak ratios $\sim 4$ to 5 are necessary at the front of the laser pulse [for a Gaussian longitudinal profile, it corresponds to a full duration at half maximum of about $(1.2 \sim 1.4) \lambda$ ].

In conclusion, we propose using a nanofilm for the generation of a single attosecond high-charge relativistic electron bunch with predefined parameters. We demon- strate the feasibility of this approach by the 2D PIC simulations. We reveal the effect of charge freezing, which guarantees supporting REM surface charge density during the evolution. We also developed the 1D self-consistent theory, which takes into account Coulomb interactions, radiation of the electrons, and laser amplitude depletion. This theory allows us to calculate dynamical parameters of the REM electrons and shows a good agreement with the results of the 2D PIC simulations. According to this theory, the initial diameter of the REM is equal to the laser beam diameter $2 w_{0}$, and the initial charge is proportional to $w_{0}^{2} \alpha$. The energy and the energy spread of the electrons in the REM are controlled by $a_{0}$ and $\alpha$, and the scaling with the laser field intensity and nanofilm thickness can be derived from Eqs. (4).

We thank the CRI Program/KOSEF of the Korean Ministry of Science and Technology for support and Professor W. Mori at UCLA for the OSIRIS code.

*Electronic address: victorvkulagin@yandex.ru Present address: APRI, GIST, Gwangju 500-712, Korea. On leave from Sternberg Astronomical Institute of MSU, Russia.

†Electronic address: hysuk@gist.ac.kr Present address: APRI, GIST, Gwangju 500-712, Korea.

[1] R. Kodama et al., Phys. Rev. Lett. 84, 674 (2000).

[2] N. Naumova et al., Phys. Rev. Lett. 93, 195003 (2004).

[3] S. D. Baton et al., Phys. Rev. Lett. 91, 105001 (2003).

[4] J. Zheng et al., Phys. Rev. Lett. 92, 165001 (2004).

[5] D. Umstadter, J. K. Kim, and E. Dodd, Phys. Rev. Lett. 76, 2073 (1996).

[6] A. G. Khachatryan, F. A. van Goor, and K.-J. Boller, Phys. Rev. ST Accel. Beams 7, 121301 (2004).

[7] N. Hafz et al., Nucl. Instrum. Methods Phys. Res., Sect. A 554, 49 (2005).

[8] G. V. Stupakov and M. S. Zolotorev, Phys. Rev. Lett. 86, 5274 (2001).

[9] V. V. Kulagin et al., Phys. Lett. A 353, 505 (2006).

[10] V. A. Cherepenin and V. V. Kulagin, Phys. Lett. A 321, 103 (2004).

[11] V. V. Kulagin, V. A. Cherepenin, and H. Suk, Appl. Phys. Lett. 85, 3322 (2004).

[12] V. V. Kulagin, V.A. Cherepenin, and H. Suk, Phys. Plasmas 11, 5239 (2004).

[13] M. Y. Shverdin et al., Phys. Rev. Lett. 94, 033904 (2005).

[14] A. Guandalini et al., J. Phys. B 39, S257 (2006).

[15] G. A. Mourou, T. Tajima, and S. V. Bulanov, Rev. Mod. Phys. 78, 309 (2006).

[16] R. G. Hemker, Ph.D. thesis, UCLA, 2000.

[17] V. A. Vshivkov et al., Phys. Plasmas 5, 2727 (1998).

[18] R. V. Volkov et al., Kvantovaya Elektron. (Moscow) 24, 1114 (1997) [Quantum Electron. 24, 1114 (1997)].

[19] B. Rau, T. Tajima, and H. Hojo, Phys. Rev. Lett. 78, 3310 (1997).

[20] K. S. Novoselov et al., Proc. Natl. Acad. Sci. U.S.A. 102, 10451 (2005). 